\title{
Maternal Separation Induced Visceral Hypersensitivity from Childhood to Adulthood
}

\author{
Lisha Yi, Haiqin Zhang, Huihui Sun, Lu Zhou, Ying Chen, Liqian Xuan, Yuanxi Jiang, and Shuchang Xu* \\ Department of Gastroenterology, Tongiji Institute of Digestive Diseases, Tongji Hospital, Tongji University School of Medicine, Shanghai, China
}

\section{Background/Aims}

Early adverse life events (EALs) are relevant to irritable bowel syndrome in adulthood. Maternal separation (MS), as one of the EALs, has proved to induce visceral hypersensitivity in adult rats. However, the effect of MS on visceral hypersensitvity from the post-weaning period to adulthood remains unknown.

\section{Methods}

One hundred and ten neonatal Sprague-Dawley rats were randomly divided into 2 groups: rats in the MS group were exposed to 3 hours daily MS on postnatal day (PND) 2-14; the normal control (NC) group remained undisturbed. Visceral sensitivity was determined by measuring the visceromotor response to colorectal distention on PND21, 35, and 56. Anxiety-like behaviors were measured by the open field test.

\section{Results}

Compared with NC rats, MS rats showed significant visceral hypersensitivity from the post-weaning period to adult. The proportion of visceral hypersensitive rats decreased with age from $87.5 \%$ to $70.0 \%$ in the female MS group and from $90.0 \%$ to $66.7 \%$ in the male MS group. The relative VMR ratio of MS and NC on PND21 was higher than PND35 and PND56. MS rats showed decreased ability of movement and exploration to the novel environment in the post-weaning period, obesity in the prepubertal period, and more anxietylike behaviors in adulthood.

\section{Conclusions}

MS can significantly affect visceral sensitivity and behaviors of rats in different age stages, especially in the post-weaning period. Visceral hypersensitivity of MS rats is more pronounced in the post-weaning period and slightly restored in adults. Thus, visceral hypersensitivity in the post-weaning period might play a more meaningful pathophysiologic role in the formation of adult irritable bowel syndrome.

(J Neurogastroenterol Motil 2017;23:306-315)

\section{Key Words}

Irritable bowel syndrome; Maternal deprivation; Visceral hypersensitivity

Received: May 30, 2016 Revised: January 1, 2017 Accepted: January 19, 2017

(a) This is an Open Access article distributed under the terms of the Creative Commons Attribution Non-Commercial License (http://creativecommons. org/licenses/by-nc/4.0) which permits unrestricted non-commercial use, distribution, and reproduction in any medium, provided the original work is properly cited.

${ }^{*}$ Correspondence: Shuchang Xu, PhD

Department of Gastroenterology, Tongji Institute of Digestive Diseases, Tongji Hospital, Tongji University School of Medicine, No. 389 Xin Cun Road, Shanghai 200065, China

Tel: +86-21-66111287, Fax: +86-21-56050191, E-mail: xsc-students@hotmail.com

Lisha Yi and Haiqin Zhang contributed equally to this work. 


\section{Introduction}

Irritable bowel syndrome (IBS), a common digestive disease associated with abdominal pain or discomfort and altered bowel habits, affects $7 \%$ to $21 \%$ of the general population. ${ }^{1}$ In recent years, early adverse life events (EALs) such as sexual, physical abuse or emotional trauma have been identified as vulnerability factors for development of adult IBS. ${ }^{2,3}$ For example, in 2009, one retrospective cohort study evaluating the epidemiology of IBS in the World War II period showed that the factor of early life exposure to severe wartime conditions was related with the increase of IBS prevalence. ${ }^{4}$ In 2012, Bradford et al ${ }^{2}$ found that IBS patients had more EALs such as common trauma, corporal punishment, emotional abuse or sexual assault than healthy subjects. Animal research has played a vital role in advances in the pathophysiology of IBS. Several studies indicated that disturbed gastrointestinal motility, visceral hypersensitivity, immune dysregulation and mucosal barrier dysfunction are thought to be involved in the development of EALs-related IBS. ${ }^{5-9}$

Visceral hypersensitivity is one important pathophysiological mechanism in the generation of IBS. ${ }^{10,11}$ Many previous experimental studies found that EALs such as maternal separation (MS) can induce adult visceral hypersensitivity. ${ }^{5,6,12}$ However, the effect of EALs on the different age stages from childhood to adulthood is not yet clear. It was found that neonatal MS could lead to the development of gastrointestinal disorders by influencing adult visceral sensitivity. ${ }^{5} \mathrm{MS}$ in rodents could well mimic EALs in humans and the MS model was commonly used to explore the underlying mechanism of EALs related IBS in many studies. ${ }^{6,7}$ Based on the MS rat model, this research aims to explore the effect of MS on visceral sensitivity and anxiety-like behavior in the different age stages of rats, and expect to provide a basis for further exploration of the mechanisms of EALs induced IBS.

\section{Materials and Methods}

\section{Animals}

One male and 3 female (sisterhood) Sprague-Dawley rats (250$350 \mathrm{~g}$ ) were obtained from Shanghai SLAC Laboratory Animal Co, LTD, China. The male rat was placed with 3 female rats for mating at the beginning of the experiment. Rats were maintained on a 12-hour light/dark cycle and provided with food and water ad libitum. After pregnancy, 3 female rats were caged separately to give birth. After rat pup weaning, the female rats were caged with the male rat in order to conceive again. All 110 newborn pups were used in this experiment. All animals were treated in accordance with the guidelines for the Care and Use of Laboratory Animals of the Animal Research Committee of the Tongji Hospital, Shanghai, China.

\section{Experimental Groups and Maternal Separation}

One hundred and ten newborn rats were randomly assigned into the MS group $(\mathrm{n}=60)$ and the normal control group (NC group, $\mathrm{n}=50$ ). Neonatal maternal separation rat model was established as reported in previous studies. ${ }^{13}$ Briefly, pups in the MS group were separated from their mothers and siblings, then placed into individual Plexiglas cages in a separate room for 180 minutes daily (10:00-13:00) from postnatal day (PND) 2 to PND $14 .{ }^{14}$ Each cage contained bedding material and a heating pad (37 \pm $0.5^{\circ} \mathrm{C}$ ) was placed under each cage to help pups regulate normal body temperature. Pups in the NC group remained undisturbed with their mothers in the cages. All pups were weaned on PND18 and well developed. Early weaning was done because the visceral motor response (VMR) of rat pups were recorded on PND21 and the abdominal surgery needed to be performed 3 days before VMR testing. After weaning, 3 pups in each group were randomly cared in one cage by sex until the VMR test. All pups were weighed at PND21, 28, 35, 42, 49 and 56.

\section{Visceral Motor Response and Electromyogram Recording}

To measure rats' visceral sensitivity, the VMR to colorectal distension (CRD) was monitored by recording electromyogram (EMG) in the post-weaning (PND21), prepubertal (PND35), and young adult (PND56) rats (experimental design showed as Fig. 1). The VMR test was performed as described in our previous study. ${ }^{15}$ Briefly, rats were anesthetized with intraperitoneal injection of pentobarbital sodium $(50 \mathrm{mg} / \mathrm{kg})$, then a pair of Teflon-coated stainless wires was surgically implanted into the left external abdominal oblique muscles 3 days before recording EMG. After surgery, the rats were housed separately until the EMG recording test. On the test day, animals were subjected to CRD. A flexible latex balloon (medical finger glove, $0.5 \mathrm{~cm}$ diameter for rats on PND21; $1.0 \mathrm{~cm}$ diameter for rats on PND35; $1.5 \mathrm{~cm}$ diameter for rats on PND56) tied around a tube (polyethylene-cannulas, $0.5 \mathrm{~mm}$ diameter for rats on PND21; intravenous tube, $2 \mathrm{~mm}$ diameter for rats on PND35 and PND56) was lubricated with liquid paraffin oil and inserted into the descending colon with the distal tip $1 \mathrm{~cm}$ from the anal verge and secured to the base of the tail under short 


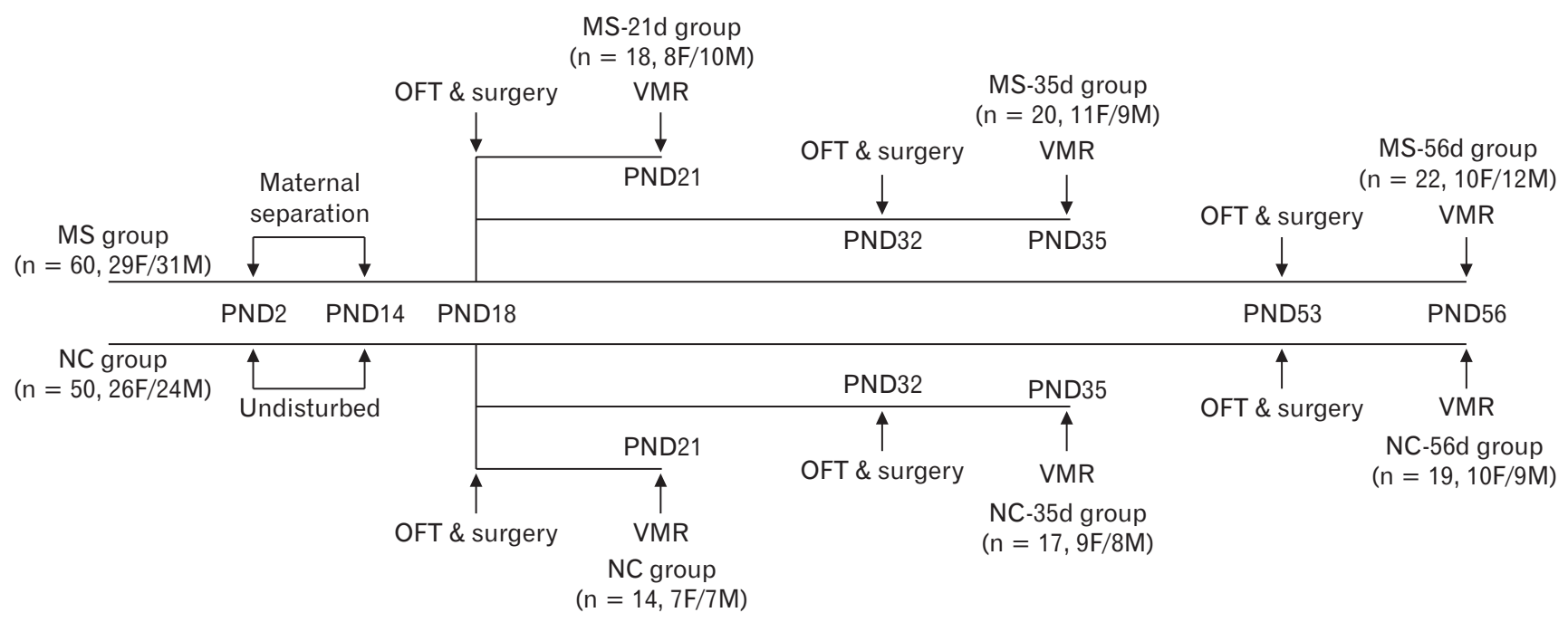

Figure 1. Experimental design. Maternal separation (MS) procedures were performed on postnatal day (PND) 2-14. After weaning, MS rats were randomly assigned into MS-21d, MS-35d, and MS-56d groups. The visceromotor response (VMR) to colorectal distension was monitored by electromyogram (EMG) recording on PND21, 35, and 56. The open field test (OFT) and surgery was performed 3 days before EMG recording. Correspondingly, the normal control (NC) group was set. The rat number of each group are shown in Figure. F, female; M, male. MS-21d, maternal separation rats on PND21.

ether anesthesia. Because of the intestinal tract similarities between rat pups and mice, a preliminary experiment was carried out to define the graded CRD protocol for post-weaning and prepubertal rats based on previous studies on the visceral hypersensitivity of mice. ${ }^{6,8}$ We found that $40 \mathrm{mmHg}$ CRD was the ECG signal detection threshold for post-weaning rats (PND21), and $20 \mathrm{mmHg}$ CRD for prepubertal rats (PND35), based on the present recording method. Thus, graded CRD (40-60-80 $\mathrm{mmHg}$ for rats on PND21; 20-40-60 mmHg for rats on PND35 and PND56) were performed by rapidly injecting gas into the colonic balloon for 1 second and maintaining the distention for 20 seconds. Three cycles of each graded CRD (20-second duration; 5-minute inter-stimulus interval) were applied to each rat. The EMG recording signal was amplified and filtered $(50-5000 \mathrm{~Hz})$ by PowerLab System (Chart 5.0; AD Instruments, Bella Vista, NSW, Australia). The results of electromyography were quantified by calculating the area under the curve per second (AUC/sec). Area under the curve is the sum of all recorded data points multiplied by the sample interval (in seconds) after baseline subtraction.

\section{Open Field Test}

All rats used in this study were subjected to the open field test (OFT) before surgery. Rats were placed in an opaque open box with black bottom and white walls $(40 \mathrm{~cm}$ length $\times 40 \mathrm{~cm}$ width $\times 40 \mathrm{~cm}$ height). The animals were placed into this arena from the center for 5 minutes. The fecal pellets were counted. All rats were transferred to the testing room 30 minutes before the experiment in order to adapt to the testing environment. The test process was recorded via a video camera above of the open field. Total travelled distance, and the number of rearing and grooming were measured by the ANY-maze system (Stoelting Co, Wood Dale, IL, USA).

\section{Statistical Methods}

All data were expressed as mean \pm SEM. Statistical analysis was performed using SPSS 17.0. Comparison between the 2 groups was analyzed by the $t$ test. Comparison among the 3 groups was analyzed by the one-way ANOVA test and within groups by the Least-significant difference test. The Fisher's exact test was used to assess the proportion of visceral hypersensitivity across the 3 different aged groups. $P<0.05$ was considered statistically significant.

\section{Results}

\section{Cut Off Value and Percentage of Hypersensitive Rats in the Maternal Separation Group}

The VMR of NC rats in 3 different periods were measured and different groups of rats were used at the different PNDs. In these 3 different periods, NC rats showed pressure-dependent increases in the VMR to CRD. Both at PND21 and PND35, 
female $\mathrm{NC}$ rats and male $\mathrm{NC}$ rats showed no remarkable difference in VMR to CRD (Fig. 2A, B). However, at PND56, the VMR to $40 \mathrm{mmHg}$ and $60 \mathrm{mmHg}$ CRD of female $\mathrm{NC}$ rats were significantly higher than that of the male $\mathrm{NC}$ rats (Fig. 2C).

In the 3 different $\mathrm{NC}$ rat periods, the $\mathrm{AUC} / \mathrm{sec}$ data of relative VMR to the highest pressure-grade $\mathrm{CRD}$ were calculated and the 90th percentile AUC/sec value was used as an arbitrary cut- off-value. These values were then used to define hypersensitivity or normal-sensitivity status in individual rats in the MS group. The $90 \% \mathrm{AUC} / \mathrm{sec}$ of female $\mathrm{NC}$ rats were 2.20 on PND21, 3.18 on PND35, and 4.15 on PND56, while the 90\% AUC/sec of male NC rats were 2.04 on PND21, 3.06 on PND35, and 3.37 on PND56.

Based on these pre-defined cut-off-values, Figure 2E shows
A

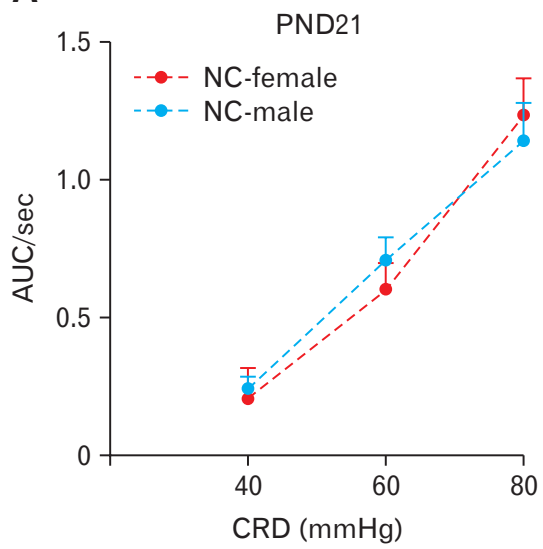

B

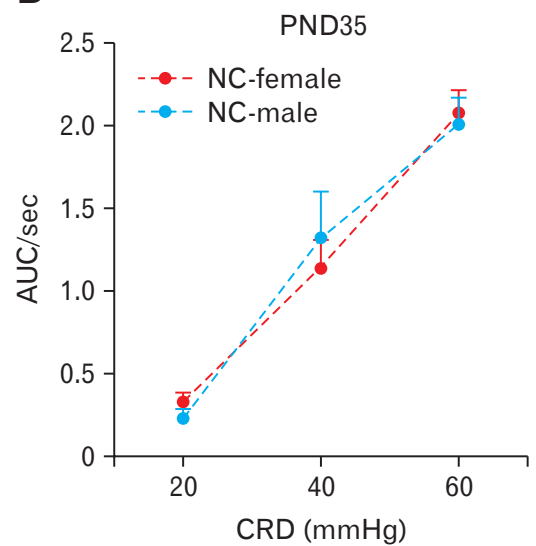

C

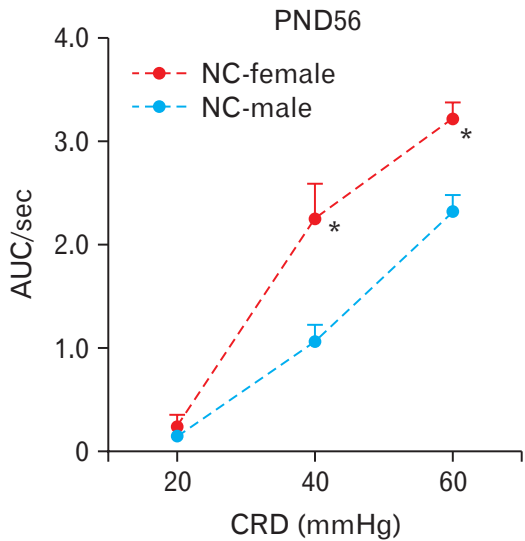

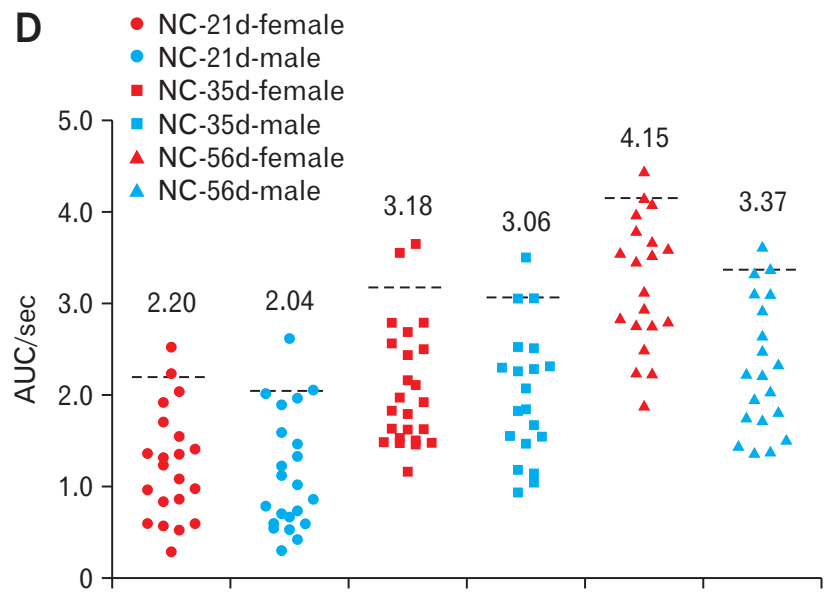
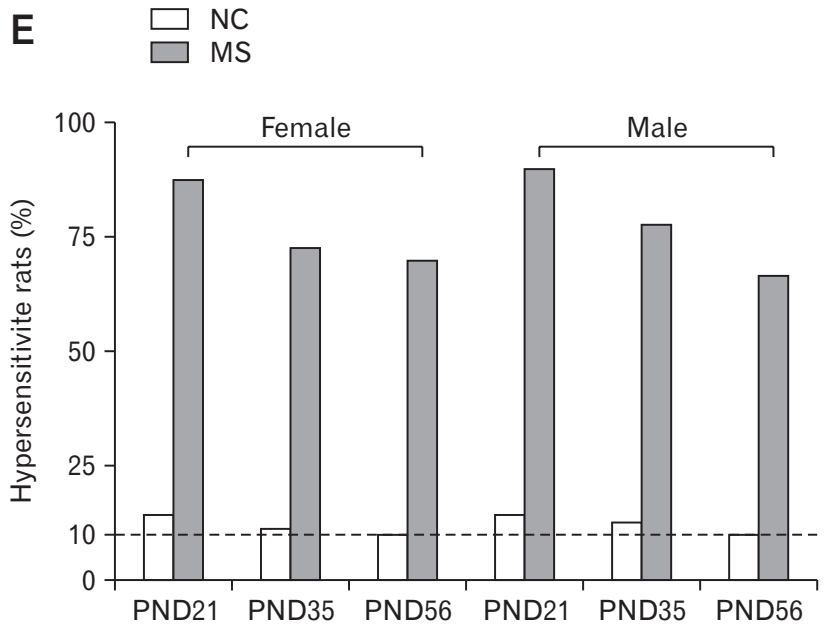

Figure 2. Visceromotor response (VMR) of normal control (NC) rats in 3 different age periods. All data (A-C) are given as mean \pm SEM. (A) The VMR of NC rats on the postnatal day (PND) 21. Female NC rats $(n=7)$ and male $\mathrm{NC}$ rats $(\mathrm{n}=7)$ showed no remarkable difference in VMR to colorectal distension (CRD) on PND21. (B) VMR of NC rats on PND35. No remarkable difference in VMR to CRD was found between female $\mathrm{NC}$ rats $(\mathrm{n}=9)$ and male $\mathrm{NC}$ rats $(\mathrm{n}=8)$ on PND35. (C) VMR of NC rats on PND56. VMR of $40 \mathrm{mmHg}$ and $60 \mathrm{mmHg}$ $\mathrm{CRD}$ of female $\mathrm{NC}$ rats $(\mathrm{n}=10)$ were significant higher than that of male $\mathrm{NC}$ rats $(\mathrm{n}=9)(40 \mathrm{mmHg}-\mathrm{CRD}: 2.2 \pm 0.69$ vs $1.06 \pm 0.32$ and $60 \mathrm{mmHg}-\mathrm{CRD}: 3.23 \pm 0.71$ vs $2.32 \pm 0.72$ ), ${ }^{*}<<0.05$. (D) The area under the curve per second (AUC/sec) data of all electromyogram (EMG) recordings for $50 \mathrm{NC}$ rats in 3 different periods. The 90th percentile AUC/sec was calculated and used as an arbitrary cut-off-value. The predefined cut-off-values of female NC rats were 2.20 on PND21, 3.18 on PND35, and 4.15 on PND56. The predefined cut-off-values of male NC rats were 2.04 on PND21, 3.06 on PND35, and 3.37 on PND56. In (E) the cut-off-values were used to define the percentage of maternal separation (MS) group hypersensitive rats in the 3 different age periods. A high percentage of visceral hypersensitive female (7/8) and male (9/10) rats were observed in the MS group on PND21. For the PND56, the percentage of hypersensitive rats reduced to 70.0\% (7/10) in female rats and $66.7 \%(8 / 12)$ in male rats. NC-21d, normal control rats on PND21. 
the percentage of visceral hypersensitive animals in the MS group in 3 different periods. By definition, 87.5\% (7/8) female and 90.0\% (9/10) male rats in the MS group were visceral hypersensitive on PND21. While on PND56, the percentage of hypersensitive rats in the MS group reduced to $70.0 \%$ (7/10) in female and $66.7 \%$ $(8 / 12)$ in male rats. Either female or male rats, the MS pups group (PND21) had a higher proportion of visceral hypersensitive rats than the MS prepubertal (PND35) and young adult (PND56) groups (female: $\chi^{2}=26.47, P<0.01$; male: $\chi^{2}=48.87, P<0.01$,
Fisher's exact test).

\section{Comparing the Visceral Sensitivity Between Normal Control and Maternal Separation Groups in Different Age Stages}

Based on the cut-off-values described in Figure 2D, the VMR data of normal-sensitive animals $(n=14)$ in the MS group were excluded. In the different age stages, the mean VMR amplitudes (AUC/sec) to CRD in both MS and NC groups showed a
A

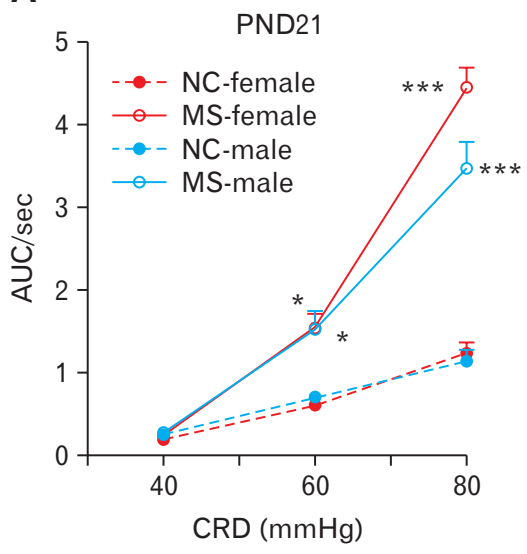

D

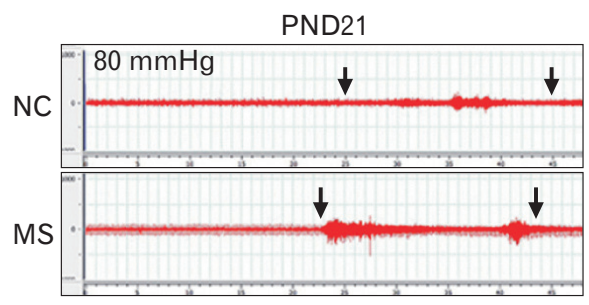

E

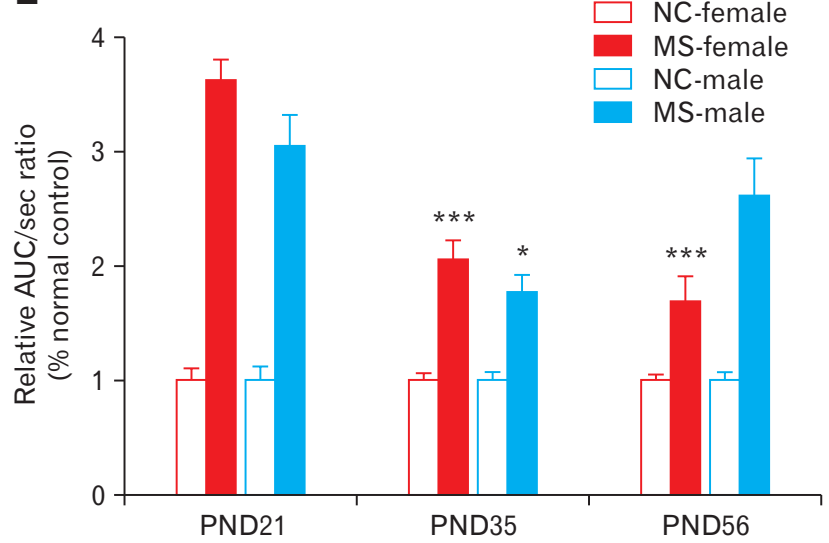

PND35

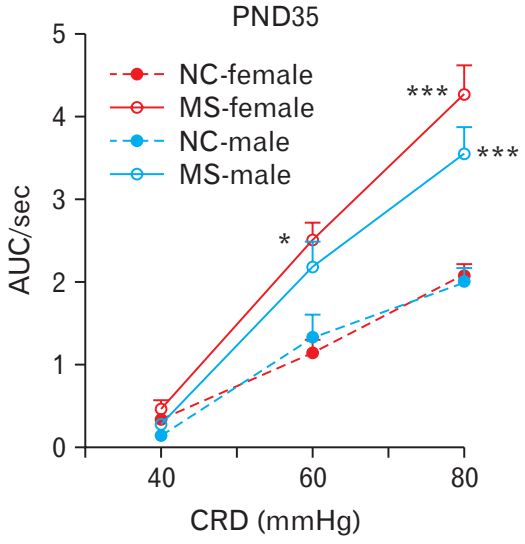

C

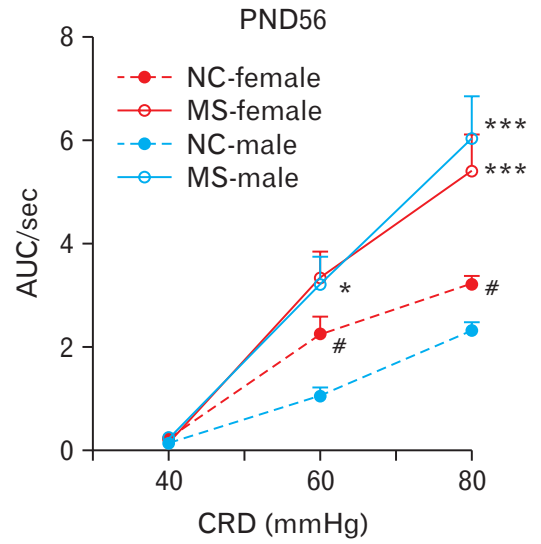

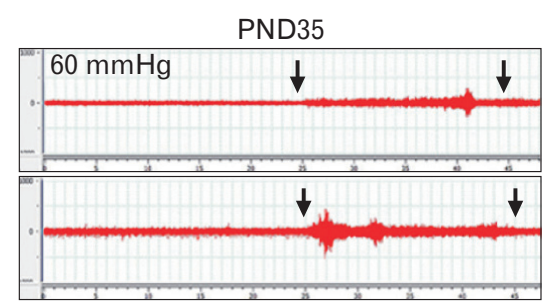

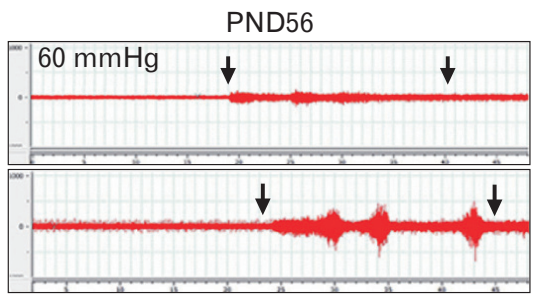

Figure 3. The visceromotor response (VMR) of maternal separation (MS) and normal control (NC) rats in the 3 different age periods. (A) On postnatal day 21 (PND21), the mean amplitudes of VMR to $60 \mathrm{mmHg}$ and $80 \mathrm{mmHg}$ colorectal distension (CRD) in the MS group were significantly higher than that of the NC group. On PND35 (B) and PND56 (C), the mean VMR amplitudes of $60 \mathrm{mmHg}$ CRD in the MS group were significantly higher than that in the NC group. (D) Representative electromyogram (EMG) recordings of MS and $\mathrm{NC}$ rats in the 3 different periods. The arrow shows the start time of distention and the start time of deflation. Results are presented as mean \pm SEM. On PND21: MS, $n=16,7 \mathrm{fe}-$ male/9 male $(7 \mathrm{~F} / 9 \mathrm{M}) ; \mathrm{NC}, \mathrm{n}=14,7 \mathrm{~F} / 7 \mathrm{M}$. On PND35: MS, $\mathrm{n}=15$, $8 \mathrm{~F} / 7 \mathrm{M} ; \mathrm{NC}, \mathrm{n}=17,9 \mathrm{~F} / 8 \mathrm{M}$. On PND56: MS, $\mathrm{n}=15,7 \mathrm{~F} / 8 \mathrm{M} ; \mathrm{NC}, \mathrm{n}$ $=19,10 \mathrm{~F} / 9 \mathrm{M} .{ }^{*} P<0.05 \mathrm{NC}$ vs MS, ${ }^{* * *} P<0.001 \mathrm{NC}$ vs $\mathrm{MS},{ }^{\#} P<$ 0.05 female vs male in the NC group. (E) The relative VMR ratios of MS and $\mathrm{NC}$ rats in different age stages. In both female and male groups, the relative VMR ratio of MS and NC rats on PND21 was higher than that of PND35. ${ }^{* *} P<0.0001$ vs MS-female on PND21, ${ }^{*} P<0.05$ vs MSmale on PND21. AUC/sec, area under the curve per second. 
pressure-dependent increase (Fig. 3A-C). Compared with female and male rats in the $\mathrm{NC}$ group, the VMR mean amplitudes of 60 $\mathrm{mmHg}$ and $80 \mathrm{mmHg} \mathrm{CRD}$ in the MS group were significantly higher in the PND21 (Fig. 3A). On both PND35 and PND56 of female and male rats, the mean VMR amplitudes of the $60 \mathrm{mmHg}$

A
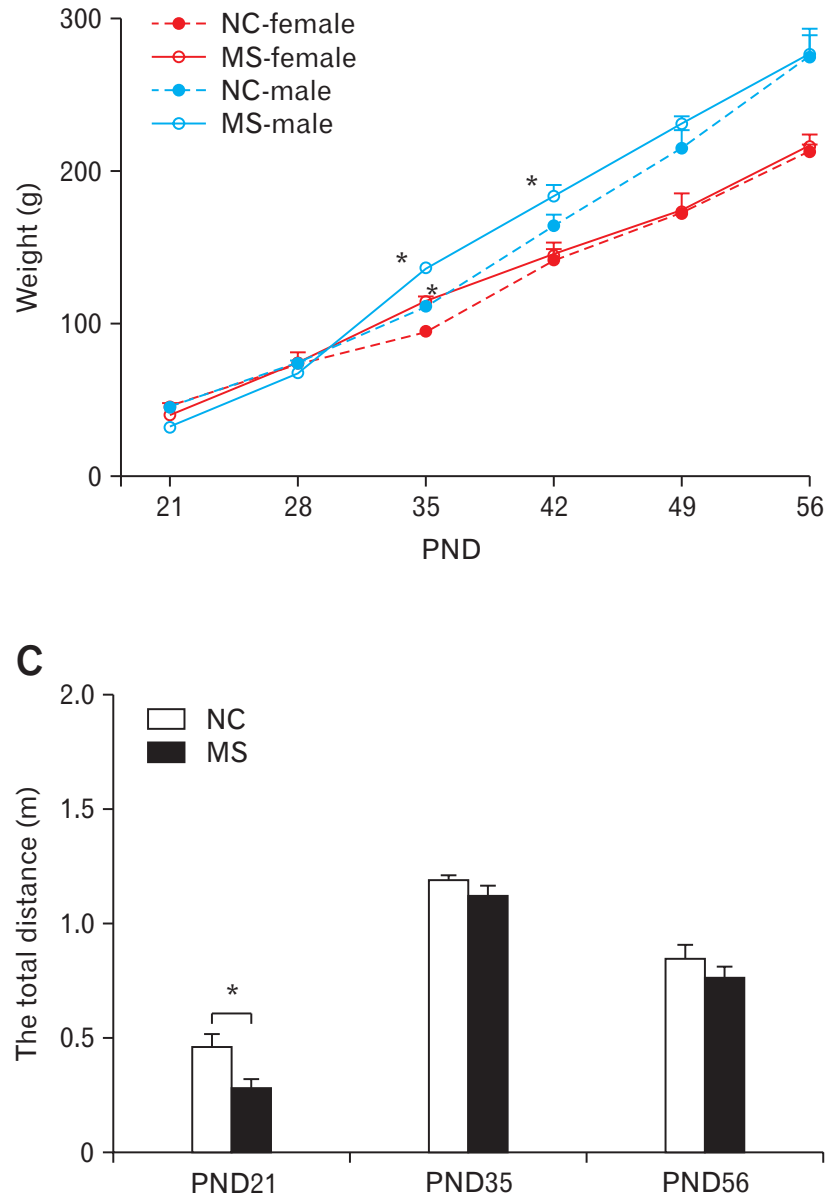

E

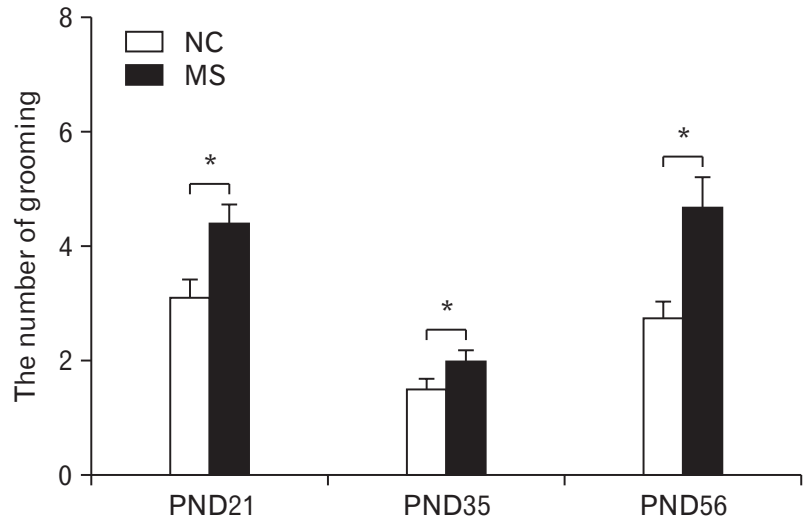

$\mathrm{CRD}$ in the MS group were significantly higher than that of the NC group (Fig. 3B and 3C). On PND56, although female NC rats showed significantly higher VMR of $40 \mathrm{mmHg}$ and $60 \mathrm{mmHg}$ CRD compared with male $\mathrm{NC}$ rats, female and male MS rats showed no markedly difference in VMR to those 3 graded-pressure

B

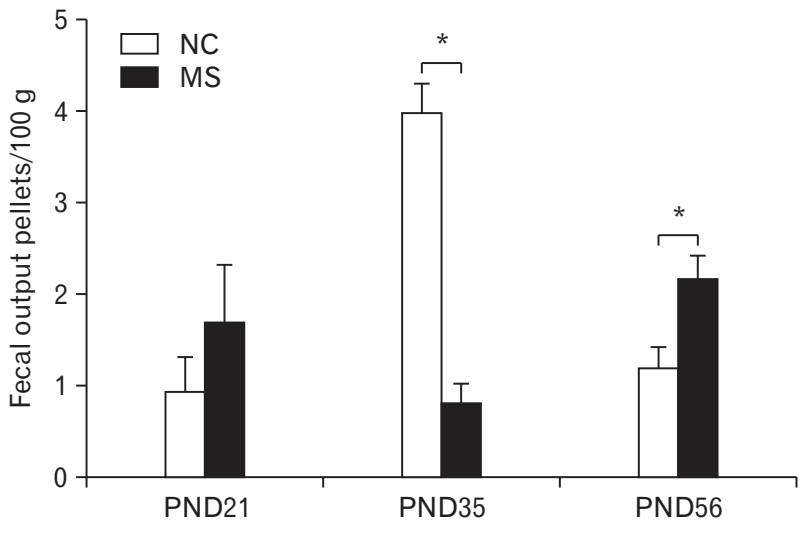

D

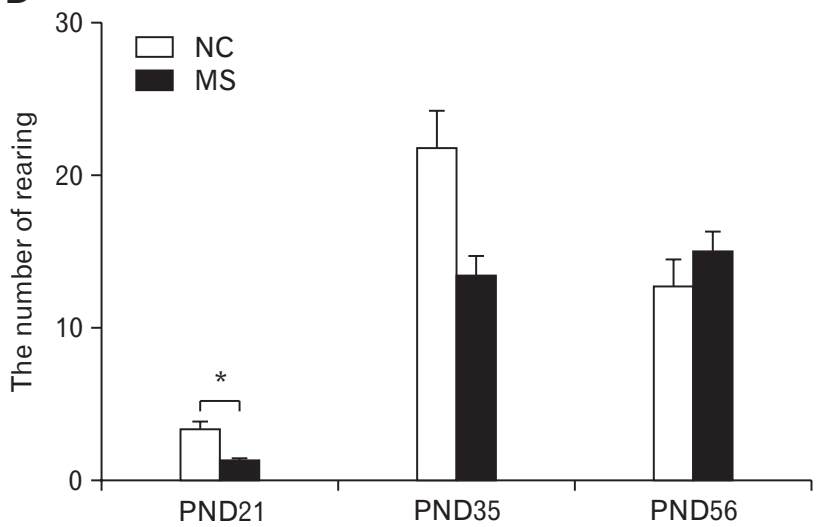

Figure 4. Weight, defecation, and anxiety behaviors in normal control (NC) and maternal separation (MS) groups. (A) The body weight of $\mathrm{NC}$ and MS rats. On postnatal day (PND) 35, the body weight of MS rats was significantly higher than that of NC pups (114.78 \pm 2.91 vs $94.64 \pm 2.84$ in female, $136.47 \pm 2.15$ vs $111.00 \pm 1.22$ in male; ${ }^{*} P<0.05$ vs NC). Before surgery, each group of rats was placed in the open field apparatus to evaluate anxiety behaviors. In the open field test, the data of both male and female rats were used together for analysis. Fecal output pellets per $100 \mathrm{~g}$ body weight (B), total distance traveled (C), number of rearing (D), and number of grooming (E) in the MS and NC groups were measured. Results were presented as mean \pm SEM. ${ }^{*} P<0.05$ vs NC. 
CRDs (Fig. 3C). Furthermore, under the highest graded-pressure $\mathrm{CRD}$, the relative VMR ratio of $\mathrm{MS}$ and $\mathrm{NC}$ rats in the 3 different periods were analyzed. Figure $3 \mathrm{E}$ depicts that the relative VMR ratio of MS and $\mathrm{NC}$ on PND21 was higher than the relative ratio on PND35 and PND56.

\section{Comparing the Weight and Defecation Between the 2 Groups in Different Age Stages}

On PND21 and PND28, the mean body weight of MS rats was lower than that of $\mathrm{NC}$ rats, but without statistical significance. On PND35, the body weight of MS rats was significantly higher than that of NC pups $(114.78 \pm 2.91$ vs $94.64 \pm 2.84$ in females, $136.47 \pm 2.15$ vs $111.00 \pm 1.22$ in males, $P<0.05)$. On PND49 and PND56, there were no significantly differences in the body weight between the MS and NC groups (Fig. 4A). Defecation between the 2 groups in different age stages showed a dynamic change: on PND21, no significant differences were found in fecal pellets per $100 \mathrm{~g}$ body weight between the MS and NC pups; on PND35, the fecal number per $100 \mathrm{~g}$ body weight of MS rats was significantly lower than that of NC rats; but on PND56, the fecal number per $100 \mathrm{~g}$ body weight of MS rats was significantly higher than that of $\mathrm{NC}$ rats (Fig. 4B).

\section{Comparing the Change of Anxiety-like Behaviors Between the 2 Groups in Different Age Stages}

Factors such as total travelled distance, rearing, and grooming, were recorded in all rats among different age stages by using the OFT. On PND21, the distance and rearing numbers in the MS group were significantly lower than that of the $\mathrm{NC}$ group $(P<0.05$; Fig. 4C and 4D). On PND35, the rearing numbers in the MS group were still significantly lower than that of the $\mathrm{NC}$ group $(P<$ 0.05; Fig. 4D), but the total travelled distance showed significant differences between the 2 groups. On PND56, the distance and rearing numbers between the MS group and NC group had no significant differences, but the grooming numbers in the MS group were significantly higher than that of the $\mathrm{NC}$ group $(P<0.05$; Fig. $4 \mathrm{E})$. The alterations in behavioral responses to OFT are shown in Figure 4C-E.

\section{Discussion}

This present study identifies for the first time that exposing rat pups to neonatal MS causes a long-lasting visceral hypersensitivity accompanied by anxiety behaviors from the post-weaning period to the young adult.
Visceral hypersensitivity is a key factor in the pathophysiology of IBS. In a variety of stress related IBS models, MS of newborn rats is considered to be a well-established model of early life stress induced visceral hypersensitivity. ${ }^{7}$ In 2002, Coutinho et $\mathrm{al}^{5}$ showed that maternal separated Long-Evans rats suffered from stressinduced visceral hyperalgesia and increased colonic motility, which could mimic IBS in humans. To date, accumulating animal experiments ${ }^{16}$ confirm that neonatal MS could induce visceral hyperalgesia in adult rats, suggesting early life adverse events can strengthen neural responses to visceral nociceptive stimulus in adulthood. Most of those reports have assessed visceral sensitivity of maternal separated rats at 10-12 weeks, ${ }^{12,17}$ which failed to clearly explain the dynamic effect of neonatal MS on visceral sensitivity of different aged rats, from the post-weaning period to adulthood.

In the present study, early life stress was modeled by MS of rats. Based on the MS model, the effect of MS on visceral sensitivity in the different age stages of rats was explored. Not all individual MS rats became visceral hypersensitive in the 3 different age periods. The proportion of visceral hypersensitive rats decreased with age from $87.5 \%$ to $70.0 \%$ in the female MS group and from $90.0 \%$ to $66.7 \%$ in the male MS group. The visceral sensitivity results also showed that MS rats exhibited significant and persistent visceral hyperalgesia from the post-weaning to adult period. Thus, neonatal MS can not only induce visceral hypersensitivity in adult rats, but also leads to little pups and prepubertal rats having a significant increased visceral sensitivity. Furthermore, we analyzed the relative VMR amplitude ratios of MS and NC rats in the different age stages. It was found that the relative VMR ratio of MS and NC rats on PND21 was significantly higher than that of PND35 and PND56, which indicated that MS-induced visceral hypersensitivity was more pronounced in the post-weaning period. One previous study about rectal sensitivity and motility in childhood IBS showed that rectal pain threshold of IBS children was significantly lower than that of the healthy control children, and all IBS children selected in this research had increased visceral hypersensitivity which occurred in only $50 \%$ adult IBS patients. ${ }^{18}$ Therefore, visceral hypersensitivity induced by EALs in childhood might play a more meaningful pathophysiologic role in the formation of adult IBS, which is worthy of attention.

The present study observed that sex differences exist in the visceral hypersensitivity of MS and NC rats. Female NC rats showed significantly higher VMR amplitudes of $40 \mathrm{mmHg}$ and 60 $\mathrm{mmHg}$ CRD compared with male NC rats on PND56, but not on PND21 and PND35. Compared with male rats on PND56, female MS rats showed a non-significant higher percentage of 
visceral hypersensitivity trend. This is consistent with a report from previous clinical studies showing sex ratio in IBS is highly skewed towards the female gender, ${ }^{19}$ which support a role of ovarian hormones. Several experimental studies found that estrogen has an activational effect on modulating visceral sensitivity, ${ }^{20}$ which could acts at multiple sites in the nerve system and facilitate spinal or supraspinal processing of visceral nociception. ${ }^{21,22}$

Through observing the changes of defecation in different periods after neonatal MS, we found that the fecal output pellets of MS rats were significantly different from NC rats on PND35 and PND56, which suggest that MS rats showed alternating diarrhea and constipation change from the prepubertal to the adult stage.

Neonatal MS also had a great impact on body weight. In this study, results showed that either male or female MS pups got lower weight during the neonatal period. However, the body weight of MS rats increased quickly and the mean weight of MS rats became higher than NC rats on PND35. Then, on PND56, the mean weight of the MS group and the NC group had no significant differences. The low body weight in MS pups' stage was possibly related with many complex factors, such as decreased breast-feeding time, lack of mother's touching, and more caloric expenditure to keep warm. The quick increase of weight in the MS adolescent stage may be associated with early MS stress. Stress is a common risk factor for obesity in children and adults. Some previous studies found that psychological stress in childhood could deteriorate lifestyle behaviors and the balance of cortisol, which may lead to the formation of adolescent obesity. ${ }^{23,24}$ Animal studies also found that early life stressful events such as MS in the rodent can cause disruption of the HPA axis. ${ }^{7,25,26}$ Early life stress can influence development of the HPA axis, as well as regulation of satiety-related hormones, leptin, insulin, and ghrelin, again influencing appetite, feeding behavior, and metabolism, which results in the development of obesity. ${ }^{27}$ The weight of MS rats can gradually return to normal level on PND56, suggesting that the harmful effect of MS on weight can be improved in rat adulthood after normal feeding.

The OFT is an experiment used to assay general locomotor activity levels and anxiety in rodents. The OFT results in this study showed that the total travelled distance and rearing number of the MS group significantly decreased on PND21. In the adult stage, the distance and rearing number in MS rats became no different with NC rats, but the grooming number in the MS group was significantly higher than $\mathrm{NC}$ rats in all 3 age periods. The distance and rearing number reflected the ability of movement and exploration to the novel environment. The grooming number reflected the state of tension and anxiety. This result suggested that MS can decrease the ability of movement and exploration to the novel environment in the post-weaning period, which can improve in the prepubertal period and basically return to normal in adulthood. The grooming number in MS rats significantly was increased suggesting that MS rats are with more tension and anxiety than $\mathrm{NC}$ rats.

Neonatal MS, as one of EALs, has a significant effect on the development of visceral hyperalgesia. It has been proven that MS could trigger a long-term alternation in the brain-gut axis, from primary afferent sensitization to the dysfunction of the endogenous pain modulation system. The sensitized cingulate cortex and upregulation of the activity of the ascending pathway at the spinal level, as well as the thalamo-cortico-amydala pathway, may be responsible for the development of visceral hypersensitivity. ${ }^{28}$ Neonatal MS induced long-term changes in neuroendocrine and neuropeptide secretion may also contribute to visceral hyperalgesia. Corticotrophin releasing factor ${ }^{29}$ and nerve growth factor ${ }^{30}$ were proved to be relevant to the neonatal MS-induced visceral hyperalgesia. In the past few years, the underlying relationship between gut microbiota and functional gastrointestinal disorders has attracted more attention. O'Mahony et $\mathrm{al}^{9}$ showed that there is a link between stress physiology and gut microbiota. Recently, van den Wijngaard et $\mathrm{al}^{31}$ found that MS-induced susceptibility to stress triggered visceral hypersensitivity could transfer across generations, and that this transfer depends on maternal care, which indicated that MS is a suitable model to evaluate environmental triggers relevant to IBS clustering in families. The mechanism of EALs induced visceral hypersensitivity from childhood to adulthood needs to be further explored. This research suggests that the MS model can be used to explore early life adverse triggers and mechanisms relevant to IBS dynamically.

In conclusion, this study showed that neonatal MS can significantly affect the weight, fecal expulsion, visceral sensitivity and behaviors in 3 different age periods. Neonatal MS could induce significant and persistent visceral hyperalgesia from the post-weaning to the adult. Also, visceral hypersensitivity in the post-weaning period might play a more meaningful pathophysiologic role in the formation of adult IBS, which is worthy of attention.

Acknowledgements: We are grateful to the Central Laboratory of Tongji Hospital, Shanghai, China.

Financial support: The research was supported by the National Nature Science Foundation of China (Grant No. 81370491 and $81570484)$. 
Author contributions: Lisha Yi and Haiqin Zhang conducted the research, analyzed the data, and wrote this manuscript; Huihui Sun and Ying Chen contributed to performing the research and wrote this manuscript; Shuchang Xu contributed to design the study and revised the paper critically; Lu Zhou and Liqian Xuan contributed to performing the research and acquiring the data; and Yuanxi Jiang contributed to analysis of the data and revised the paper critically.

\section{References}

1. Chey WD, Kurlander J, Eswaran S. Irritable bowel syndrome: a clinical review. JAMA 2015;313:949-958.

2. Bradford K, Shih W, Videlock EJ, et al. Association between early adverse life events and irritable bowel syndrome. Clin Gastroenterol Hepatol 2012;10:385-390, e1-e3.

3. Chitkara DK, van Tilburg MA, Blois-Martin N, Whitehead WE. Early life risk factors that contribute to irritable bowel syndrome in adults: a systematic review. Am J Gastroenterol 2008;103:765-774.

4. Klooker TK, Braak B, Painter RC, et al. Exposure to severe wartime conditions in early life is associated with an increased risk of irritable bowel syndrome: a population-based cohort study. Am J Gastroenterol 2009;104:2250-2256.

5. Coutinho SV, Plotsky PM, Sablad M, et al. Neonatal maternal separation alters stress-induced responses to viscerosomatic nociceptive stimuli in rat. Am J Physiol Gastrointest Liver Physiol 2002;282:G307-G316.

6. Moloney RD, O'Leary OF, Felice D, Bettler B, Dinan TG, Cryan JF. Early-life stress induces visceral hypersensitivity in mice. Neurosci Lett 2012;512:99-102.

7. Barreau F, Ferrier L, Fioramonti J, Bueno L. New insights in the etiology and pathophysiology of irritable bowel syndrome: contribution of neonatal stress models. Pediatr Res 2007;62:240-245.

8. La JH, Gebhart GF. Condition-specific role of colonic inflammatory molecules in persistent functional colorectal hypersensitivity in the mouse. Neurogastroenterol Motil 2014;26:1730-1742.

9. O'Mahony SM, Marchesi JR, Scully P, et al. Early life stress alters behavior, immunity, and microbiota in rats: implications for irritable bowel syndrome and psychiatric illnesses. Biol Psychiatry 2009;65:263-267.

10. Kanazawa M, Hongo M, Fukudo S. Visceral hypersensitivity in irritable bowel syndrome. J Gastroenterol Hepatol 2011;26(suppl 3):119-121.

11. Farzaei MH, Bahramsoltani R, Abdollahi M, Rahimi R. The role of visceral hypersensitivity in irritable bowel syndrome: pharmacological targets and novel treatments. J Neurogastroenterol Motil 2016;22:558-574.

12. Bian ZX, Zhang M, Han QB, Xu HX, Sung JJ. Analgesic effects of JCM-16021 on neonatal maternal separation-induced visceral pain in rats. World J Gastroenterol 2010;16:837-845.

13. Tjong YW, Ip SP, Lao L, et al. Role of neuronal nitric oxide synthase in colonic distension-induced hyperalgesia in distal colon of neonatal maternal separated male rats. Neurogastroenterol Motil 2011;23:666, e278.

14. Wouters MM, Van Wanrooy S, Casteels C, et al. Altered brain activation to colorectal distention in visceral hypersensitive maternal-separated rats. Neurogastroenterol Motil 2012;24:678, e297.

15. Yi L, Sun $H, G e C$, et al. Role of insular cortex in visceral hypersensitivity model in rats subjected to chronic stress. Psychiatry Res 2014;220:11381143.

16. Ren TH, Wu J, Yew D, et al. Effects of neonatal maternal separation on neurochemical and sensory response to colonic distension in a rat model of irritable bowel syndrome. Am J Physiol Gastrointest Liver Physiol 2007;292:G849-G856.

17. Welting O, Van Den Wijngaard RM, De Jonge WJ, Holman R, Boeckxstaens GE. Assessment of visceral sensitivity using radio telemetry in a rat model of maternal separation. Neurogastroenterol Motil 2005;17:838845 .

18. Van Ginkel R, Voskuijl WP, Benninga MA, Taminiau JA, Boeckxstaens GE. Alterations in rectal sensitivity and motility in childhood irritable bowel syndrome. Gastroenterology 2001;120:31-38.

19. Meleine M, Matricon J. Gender-related differences in irritable bowel syndrome: potential mechanisms of sex hormones. World J Gastroenterol 2014;20:6725-6743.

20. Chaloner A, Greenwood-Van Meerveld B. Sexually dimorphic effects of unpredictable early life adversity on visceral pain behavior in a rodent model. J Pain 2013;14:270-280.

21. Ji Y, Tang B, Traub RJ. Spinal estrogen receptor alpha mediates estradiol-induced pronociception in a visceral pain model in the rat. Pain 2011;152:1182-1191.

22. Hubbard CS, Karpowicz JM, Furman AJ, da Silva JT, Seminowicz DA, Traub RJ. Estrogen-dependent visceral hypersensitivity following stress in rats: an fMRI study. Mol Pain 2016;12:1-10.

23. Essex MJ, Klein MH, Cho E, Kalin NH. Maternal stress beginning in infancy may sensitize children to later stress exposure: effects on cortisol and behavior. Biol Psychiatry 2002;52:776-784.

24. Miller GE, Chen E, Parker KJ. Psychological stress in childhood and susceptibility to the chronic diseases of aging: moving toward a model of behavioral and biological mechanisms. Psychol Bull 2011;137:959-997.

25. Gareau MG, Jury J, MacQueen G, Sherman PM, Perdue MH. Probiotic treatment of rat pups normalises corticosterone release and ameliorates colonic dysfunction induced by maternal separation. Gut 2007;56:1522-1528.

26. Gareau MG, Silva MA, Perdue MH. Pathophysiological mechanisms of stress-induced intestinal damage. Curr Mol Med 2008;8:274-281.

27. Sominsky L, Spencer SJ. Eating behavior and stress: a pathway to obesity. Front Psychol 2014;5:434.

28. Chung EK, Zhang X, Li Z, Zhang H, Xu H, Bian Z. Neonatal maternal separation enhances central sensitivity to noxious colorectal distention in rat. Brain Res 2007;1153:68-77.

29. Schwetz I, McRoberts JA, Coutinho SV, et al. Corticotropin-releasing factor receptor 1 mediates acute and delayed stress-induced visceral hyperalgesia in maternally separated Long-Evans rats. Am J Physiol Gastrointest Liver Physiol 2005;289:G704-G712.

30. Chung EK, Zhang XJ, Xu HX, Sung JJ, Bian ZX. Visceral hyperalgesia induced by neonatal maternal separation is associated with nerve growth factor-mediated central neuronal plasticity in rat spinal cord. Neurosci- 
ence 2007;149:685-695

31. van den Wijngaard RM, Stanisor OI, van Diest SA, et al. Susceptibility to stress induced visceral hypersensitivity in maternally separated rats is transferred across generations. Neurogastroenterol Motil 2013;25:e780e790. 